\title{
Entanglement and teleportation via chaotic system
}

\author{
N. Metwally ${ }^{1}$, L. Chotorlishvili ${ }^{2,3}$, V. Skrinnikov ${ }^{3}$ \\ 1 Math. Dept., College of Science, Bahrain University, 32038 Bahrain. \\ ${ }^{2}$ Institute for Physik, Universität Augsburg, 86135 Augsburg, Germany \\ ${ }^{3}$ Physics Department of the Tbilisi State University, Chavchavadze av.3, 0128, \\ Tbilisi
}

\begin{abstract}
The dynamics of entangled state interacting with a single cavity mode is investigated in the presence of a random parameter. We have shown that degree of entanglement decays with time and rate of decay is defined by features of random parameter. Quantum teleportation through dissipative channal and teleportation fidelity as a function of damping rates has been studied. The sensitivity of the fidelity with respect to random parameter is discussed. We have evaluated the time interval during which one can perform the quantum teleportation and send the information with reasonable fidelity, for a given values of correlation length of random parameter.
\end{abstract}

Keywords: Quantum chaos, Cavity quantum electrodynamic, Quantum information processing.

\section{Introduction}

Entanglement is a promising resource for the quantum information and communication fields [1]. There are much attentions have been paid to the manipulation of quantum entanglement[2]. Quantum teleportation [3] and quantum cryptography [4, 5] all rely on having entangled states of qubits. Therefore generating entangled states and keeping them survival for along time is an important task [6]. In the real word, quantum entanglement will be inevitably affected by the deocherence, which causes deterioration of entanglement [7]. Thus, there are several studies which concentrate on investigating the dynamics of quantum entanglement and information in open systems (see for example [8, 9]). Also, the thermal effect on the dynamic of the information has been treated in many different systems. As an example, the effect of the local thermal and squeezed reservoirs on the degree of entanglement is investigated by Ikram et. al [10]. The entanglement decay and the sudden death in the presences of thermal reservoir is discussed by Zhou and et. al [11]. The dynamics of quantum information is investigated by means of the information exchange and 
the disturbance in 12 .

Entanglement affected by other factors than thermal reservoirs. For example, system may contain stochastic parameter averaging with respect to which leads to the de-coherence. Problems like this are typical for Cavity Quantum Electrodynamics (CQED). Modern experiments in CQED have achieved strong atom-field coupling for the strength of the coupling exceeds decay process. If so, then dynamic of two level atoms inside a cavity is reduced to the driven Jaynes-Cummings (JC) Hamiltonian, which models the interaction of a single mode of an optical cavity having resonant frequency with a two level atom [13, 14]. However as was shown in [15], in the most general case dynamics is very complicated and chaotic. Since it involves not only the internal atomic transitions and field states, but also center-ofmass motion of the atom and recoil effect. Motion of two level optical atoms inside a cavity can be chaotic [15]. So, the coordinate of the atom is a random parameter and leads to the sufficient influence on the quantum information processing.

It is well-known that, in a perfect scheme, the shared entangled state is a maximally entangled state enabling perfect quantum state transfer. However interaction with the environment results in a loss of coherence. This phenomenon is key issue of many studies [8, 9]). However, the main idea of the given paper is to study the entangled states in the presence of inner random parameter in the system. For this purpose, we shall consider a generalized JC model introduced in [16]. Also, the possibility of performing quantum teleportation through this chaotic system will be discussed. Study of the chaotic quantum systems is a striking problem of quantum chaos. In the semiclassical limit quantum chaotic systems display properties similar to the classical chaos. For example, decay rate of the quantum Loschmidt echo is related to the classical Lyapunov exponent [17, 18, 19, 20]. However problem of our interest is purely of quantum origin. Since we are interested in the consequences of quantum chaos in quantum information processing.

This letter is organized as follows: In Sec.2, we describe the system and its solution. Sec.3, is devoted to investigate the dynamics of entanglement. In Sec.4, The fidelity of the teleported state is discussed. Finally, we summarize our results in Sec.5. 


\section{The Model}

During the last decade many theoretical and experimental efforts have been done in order to study processes involving atoms inside a cavity, stimulated by the experimental realization of a multi-photon micromaser [21]. In the rotating wave approximation, the interaction of the cavity mode with the injected atoms is described by the Hamiltonian [16],

$$
\begin{aligned}
\mathcal{H} & =\omega_{0}\left({\hat{S_{1}}}^{z}+{\hat{S_{2}}}^{z}\right)+\Omega\left({\hat{S_{1}}}^{+}{\hat{S_{2}}}^{-}+{\hat{S_{2}}}^{+}{\hat{S_{1}}}^{-}\right)+\omega_{f} \hat{b}^{\dagger} \hat{b} \\
& -g_{0} \cos \left(k_{f} \hat{x}\right)\left(\left({\hat{S_{1}}}^{+}+{\hat{S_{2}}}^{+}\right) \hat{b}+\left({\hat{S_{1}}}^{-}+{\hat{S_{2}}}^{-}\right) \hat{b}^{\dagger}\right)
\end{aligned}
$$

where, the first term is local spin part, the second represents the interaction between the two spins, the third is the field cavity mode Hamiltonian and the last term is the interaction between the field and the two atoms. The operators $\hat{S}_{i}^{ \pm}$and $\hat{S}_{i}^{z}, i=1,2$ are the usual raising (lowering) and inversion operators for the two atoms respectively. Theses operators satisfy the relations,

$$
\left[\hat{S}_{i}^{z}, \hat{S}_{i}^{ \pm}\right]= \pm 2 \hat{S}_{i}^{ \pm} \delta_{i j}, \quad\left[\hat{S}_{i}^{+}, \hat{S}_{i}^{-}\right]=\hat{S}_{i}^{z} \delta_{i j}
$$

where, $\delta_{i j}=1$ if $i=j$ and zero otherwise. The constants $\omega_{0}, \omega_{f}$ are the atoms, the field frequency respectively, while $\Omega$ represents the coupling constant between the two spins.

The term $g_{0} \cos \left(k_{f} \hat{x}\right)$ is coupling between the atoms and the field, where it depends on the position of the atoms inside the cavity. This dependence serves as a source of complexity of the dynamics. As was shown in [16], time dependence $x(t)$ mimics all feature or random Wiener process. The correlation length of random variable $x(t)$ scales with kinetic energy of the atom on the input and for very slow initial conditions dynamic is close to regular. Since the motion of the adiabatic motion system is integrable, then the de-coherence time of the system can be scaled by proper choice of initial kinetic energy of the atom (see Ref [15] for example).

In the invariant sub-space of the global system, we can consider a set of complete basis of the qubit-field system as $|e e, n-1\rangle,|e g, n\rangle,|g e, n\rangle$ and $|g g, n+1\rangle$. Assume that the field is initially prepared in a coherent state, $|\psi(0)\rangle_{f}=\sum_{n=0}^{\infty} W_{n}|n\rangle$. For the atomic system, we consider the initial states of the first and second atoms are, $|\psi(0)\rangle_{1}=a_{1}|g\rangle_{1}+b_{1}|e\rangle_{1},|\psi(0)\rangle_{2}=a_{2}|g\rangle_{2}+b_{2}|g\rangle_{2}$ respectively, where 1 stands for the first atom and 2 for the second atom, with $\left|a_{i}\right|^{2}+\left|b_{i}\right|^{2}=1, i=1,2$. So, the 
initial state of the atomic system can be written as

$$
\left.\left|\psi_{12}(0)\right\rangle=C_{00}|g g\rangle+C_{01}|g e\rangle+C_{10}|e g\rangle+C_{11}|e e\rangle\right)
$$

and consequently the state of the total system is given by,

$$
\left|\psi_{s}(0)\right\rangle=\sum_{n=0}^{\infty} W_{n}\left\{|n\rangle \otimes\left(C_{00}(0)|g g\rangle+C_{01}(0)|g e\rangle+C_{10}(0)|e g\rangle+C_{11}(0)|e e\rangle\right)\right\}
$$

where, $C_{00}(0)=a_{1} a_{2}, C_{10}(0)=a_{1} b_{2}, C_{01}(0)=a_{2} b_{1}, c_{11}(0)=b_{1} b_{2}, W_{n}=\frac{\bar{n}^{n}}{\sqrt{n !}} \exp \left(-\frac{1}{2}|\bar{n}|^{2}\right)$ and $\bar{n}$ is the mean photon number inside the cavity. At any time $t>0$, the state of the atomic system and the field is given by

$$
\left|\psi_{s}(t)\right\rangle=\sum_{n=0}^{\infty}\left\{A_{n}(t)|g g, n+1\rangle+B_{n}(t)|g e, n\rangle+C_{n}(t)|e g, n\rangle+D_{n}(t)|e e, n-1\rangle\right\}
$$

where,

$$
\begin{aligned}
A_{n}(t)= & W_{n}\left\{\frac{\sqrt{n+1}}{2 \sqrt{2}(2 n+1)} e^{-i \Omega t}\left(C_{00}(0) Q[\omega(t)]-C_{01}(0) Q^{-1}[\omega(t)]\right)\right. \\
& \left.+\frac{e^{-i \Omega t}}{2 \sqrt{n+1}}\left(C_{11}(0)-\frac{1}{2 \sqrt{2}(2 n+1)}\left(C_{00}(0)-C_{01}(0)\right)\right)\right\} \\
B_{n}(t)= & W_{n}\left\{\frac{e^{-i \Omega t}}{4 \sqrt{2 n+1}}\left(C_{00}(0) Q[\omega(t)]-C_{01}(0) Q^{-1}[\omega(t)]\right)-\frac{C_{10}(0)}{2} e^{i \Omega t}\right\} \\
C_{n}(t)= & W_{n}\left\{\frac{e^{-i \Omega t}}{4 \sqrt{2 n+1}}\left(C_{00}(0) Q[\omega(t)]-C_{01}(0) Q^{-1}[\omega(t)]\right)+\frac{C_{10}(0)}{2} e^{i \Omega t}\right\} \\
D_{n}(t)= & W_{n}\left\{\frac{\sqrt{n}}{2 \sqrt{2}(2 n+1)} e^{-i \Omega t}\left(C_{00}(0) Q[\omega(t)]-C_{01}(0) Q^{-1}[\omega(t)]\right)\right. \\
& \left.-\frac{e^{-i \Omega t}}{2 \sqrt{n+1}}\left(C_{11}(0)-\frac{1}{2 \sqrt{2}(2 n+1)}\left(C_{01}(0)-C_{00}(0)\right)\right)\right\}
\end{aligned}
$$

and

$$
Q[\omega(t)]=e^{i \int_{0}^{1} \omega \grave{(t)} d \grave{t}} \text { with } \omega(t)=\sqrt{2(2 n+1)} g_{0} \cos \left(k_{f} x(t)\right), Q^{-1}[\omega(t)]=Q^{*}[\omega(t)] .
$$

Due to the chaotic motion of atoms inside of cavity, we consider the random parameter, $x(t)$ as a classical chaotic function. Therefore $Q[\omega(t)]$ should be averaged over all possible realizations of the random parameter $\omega(t)$. In this treatment, we consider the case of weak chaos, corresponding to the large values of correlation time $\tau_{c}$ of random variable $x(t)$ [16].

Since we are interested in discussing some properties of the atomic system, we calculate the density matrix of the two atoms by tracing out the field i.e $\varrho_{12}=$ 

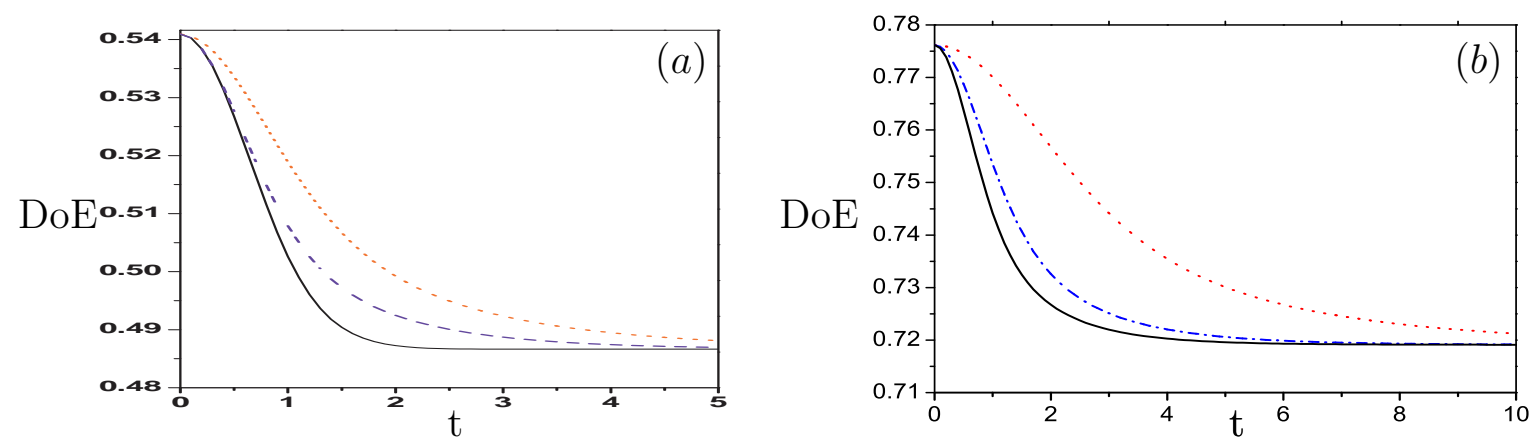

Figure 1: The degree of entanglement between two atoms initially prepared in the state (3) with $C_{00}=0.2, C_{01}=C_{10}=0$ and $C_{11}=\sqrt{1-C_{00}^{2}}$. The chaotic parameter, $\gamma=0.1,0.5$ and 0.9 , for the solid dash and dot curves respectively (a) $\bar{n}=5$ (b) $\bar{n}=6$.

$\operatorname{tr}_{f}\left\{\varrho_{A f}\right\}$, where $\varrho_{A f}=\left|\psi_{s}(t)\right\rangle\left\langle\psi_{s}(t)\right|$ and $\varrho_{12}$ is the density operator of the atomic system. In an explicit form,

$$
\begin{aligned}
\varrho_{12}(t)= & |g g\rangle \sum_{n=0}^{\infty}\left(\left|A_{n}\right|^{2}\langle g g|+A_{n} B_{n+1}^{*}\langle g e|+A_{n} C_{n+1}^{*}\langle e g|+A_{n} D_{n+2}^{*}\langle e e|\right) \\
& +|g e\rangle \sum_{n=0}^{\infty}\left(B_{n} A_{n-1}^{*}\left\langle\left. g g|+| B_{n}\right|^{2}\langle g e|+B_{n} C_{n}^{*}\langle e g|+B_{n} D_{n+1}^{*}\langle e e|\right)\right. \\
& +|e g\rangle \sum_{n=0}^{\infty}\left(C_{n} A_{n-1}^{*}\langle g g|+C_{n} B_{n}^{*}\left\langle\left. g e|+| C_{n}\right|^{2}\langle e g|+C_{n} D_{n+1}^{*}\langle e e|\right)\right. \\
& +|e e\rangle \sum_{n=0}^{\infty}\left(D_{n} A_{n-2}^{*}\langle g g|+D_{n} B_{n-1}^{*}\langle g e|+D_{n} C_{n}^{*}\left\langle\left. e g|+| D_{n}\right|^{2}\langle e e|\right) .\right.
\end{aligned}
$$

Having the density operator, we can investigate all the physical properties of the atomic system.

\section{Entanglement}

To quantify the amount of entanglement contained in the entangled states, we shall use a measurement called negativity introduced first by K. Zyczkowski [22]. This measure states that if the eigenvalues of the partial transpose are given by $\mu_{i} ; i=$ 
$1,2,3,4$, then the degree of entanglement, DOE is given by,

$$
D o E=\sum_{i=1}^{4} \mu_{i}-1,
$$

where, $\mu_{i}$ are the eigenvalues of the partial transpose of the density operator $\rho_{12}^{\mathrm{T}_{2}}(t)$.

To see the effect of the random parameter on the dynamics of entanglement, we should average the random funstion $Q[\omega(t)]$ over all possible realizations of $x(t)$ as [16],

$$
\langle Q[\omega(t)]\rangle=\exp \left[-\frac{t}{2} \sqrt{\pi \gamma} \operatorname{Erf}(t \sqrt{\gamma})\right] .
$$

Now, investigating the effect of the function $x(t)$ on the dynamics of entanglement is equivalent to investigate the effect of the parameter $\gamma$.

Fig.(1), shows the dynamics of entanglement for different values of the chaotic parameter $\gamma$, where we assume that the atomic system is initially prepared in a partial entangled state $\varrho_{12}=0.2|g g\rangle\langle g g|+\sqrt{0.6}| e e\rangle\langle e e|$. In Fig.(1a), we consider the mean photon numbers inside the cavity $\bar{n}=5$. it is clear that, for small values of the parameter $\gamma$, the entanglement decays smoothly. However, for large values of $\gamma$, the DOE, decays faster and then become stable. This means that even if the system suffers from the chaotic environment the entangle stated is robust. In Fig.(1b), describes the dynamics of entanglement for $\bar{n}=6$. One can notice that the behavior of the entanglement is the same as that depicted in Fig.(1a), namely, the entanglement decays fast as one increases the chaotic parameter $\gamma$. From Fig.(1a\&1b), it is clear that, as one increases the $\bar{n}$ inside the cavity, the entanglement improved. This behavior of entanglement is similar to that depicted in [23], where the entropy increases as the time increases. Also, due to the damping effect the oscillations are very small and appears only for a long range of time. This explain the disappearance of oscillation behavior of the entanglement.

So, one concludes then, the entanglement decays faster as one increases the chaotic parameter. Also, this effect does not cause a death of entanglement. This means that, the traveling partial entangled state in the chaotic cavity is robust. However, by increasing the mean photon numbers inside the cavity, one can delay the decay and enhance the degree of entanglement. 


\section{Teleportation}

Teleportation is one of the most promising candidate in quantum communation theory [1]. Since, it has been discovered by Bennett et. al [3], there are different protocols have been developed in different directions. Performing quantum teleportation by using entangled atoms has been investigated by many authors [25, 26, 27]. One of the most practical application is quantum teleportation through noise channels( see for example[28, 30, 29, 31]).

In this study, we investigate the effect of the chaotic parameter $\gamma$, on the fidelity of the teleported state. Let us assume that we have two users, Alice and Bob share an entangled state given by (17). Alice is given unknown state $\rho_{u}=\left|\psi_{u}\right\rangle\left\langle\psi_{u}\right|$ where,

$$
\left|\psi_{u}\right\rangle=\alpha|g\rangle+\beta|e\rangle \text { with }|\alpha|^{2}+|\beta|^{2}=1
$$

The aim of Alice is sending this unknown state to Bob, where they use the original teleportation protocol [3].

To achieve this task, the partners follow the following steps:

1. Alice performs the CNOT gate on her qubit and the given unknown qubit followed by Hadamard gate.

2. Alice measures her qubit and the unknown qubit randomly in one of the basis $\left|\phi^{ \pm}\right\rangle=\frac{1}{\sqrt{2}}(|e e\rangle \pm|g g\rangle),\left|\psi^{ \pm}\right\rangle=\frac{1}{\sqrt{2}}(|e g\rangle \pm|g e\rangle)$, and sends her results to Bob by using classical channel.

3. As soon as Bob receives the classical data from Alice, he applies a single qubit operation on his qubit depending on Alice's results.

If, Alice measures the Bell state, $\left|\phi^{+}\right\rangle=\frac{1}{\sqrt{2}}(|e e\rangle+|g g\rangle)$, then the density operator on Bob's hand, $\rho_{b}$, is given by,

$$
\rho_{b}=\kappa_{1}|g\rangle\left\langle g\left|+\kappa_{2}\right| g\right\rangle\left\langle e\left|+\kappa_{3}\right| e\right\rangle\left\langle g\left|+\kappa_{4}\right| e\right\rangle\langle e|,
$$


where,

$$
\begin{aligned}
& \kappa_{1}=\frac{1}{2} \sum_{n=0}^{\infty}\left\{|\alpha|^{2}\left|A_{n}\right|^{2}+\alpha \beta^{*} A_{n} C_{n+1}^{*}+\beta \alpha^{*} C_{n} A_{n-1}^{*}+|\beta|^{2}\left|C_{n}\right|^{2}\right\}, \\
& \kappa_{2}=\frac{1}{2} \sum_{n=0}^{\infty}\left\{|\alpha|^{2} A_{n} B_{n+1}^{*}+\alpha \beta^{*} A_{n} D_{n+2}^{*}+\beta \alpha^{*} c_{n} B_{n}^{*}+|\beta|^{2} C_{n} D_{n+2}^{*}\right\}, \\
& \kappa_{3}=\frac{1}{2} \sum_{n=0}^{\infty}\left\{\left(|\alpha|^{2} B_{n} A_{n-1}^{*}+\alpha \beta^{*} B_{n} C_{n}^{*}+\beta \alpha^{*} D_{n} A_{n-2}^{*}+|\beta|^{2} D_{n} C_{n}^{*}\right\},\right. \\
& \kappa_{4}=\frac{1}{2} \sum_{n=0}^{\infty}\left\{\left(|\alpha|^{2}\left|B_{n}\right|^{2}+\alpha \beta^{*} B_{n} D_{n+1}^{*}+\beta \alpha^{*} D_{n} B_{n-1}^{*}+|\beta|^{2}\left|D_{n}\right|^{2}\right\} .\right.
\end{aligned}
$$

To quantify the closeness of the initial state (10) with the final state (11), we evaluate the fidelity $\mathcal{F}$ which is given by,

$$
\mathcal{F}=\operatorname{tr}\left\{\rho_{u} \rho_{b}\right\}
$$

The dynamics of the fidelity $\mathcal{F}$, of the teleported state (10) is presented in Fig.(2). This figure displays the possibility of using the state $\varrho_{12}$ as a quantum channel for a short time to implement the quantum teleportation protocol. It is clear that, in the case of ideal environment namely $\gamma=0$, Alice can send the unknown state (10) with high fidelity. However for small value of the chaotic parameter $\gamma$, the fidelity $\mathcal{F}$ decreases. As one increases the chaotic parameter more, one can see that the fidelity decreases fast and at $\gamma=1$, the fidelity is zero. This behavior due to the lose of entanglement as one increases the chaotic parameter

A visible behavior of the fidelity is given in Fig.(3), where it describes $\mathcal{F}$ as a contour lines. From this figure, we can determine the interval of the critical time $\tau_{c}$, after which the effect of the chaotic parameter appears. It is easily seen that, the teleported state can be transmitted with high fidelity from one location to another for any value of the chaotic parameter $\gamma$ in the interval $t \in[0,0.25]$. However, for small values of $\gamma \in[0,0.14]$, one can teleporte the information safely with $\mathcal{F}=1$.

For $t>0.25$, the fidelity decays smoothly and sharply for larger time. As one increases $\gamma$ more, the fidelity decreases smoothly and then becomes constant. This behavior is seen for any value of the chaotic parameter $\gamma$. Also, one can see that as one increases the chaotic parameter, the dark sectors increases. This means that the fidelity decreases. However the bright regions increases as one decreases the value of the chaotic parameter.

From theses results, one can say that it is possible to achieve quantum teleportation for any value of the chaotic parameter with reasonable fidelity. Also, for small 


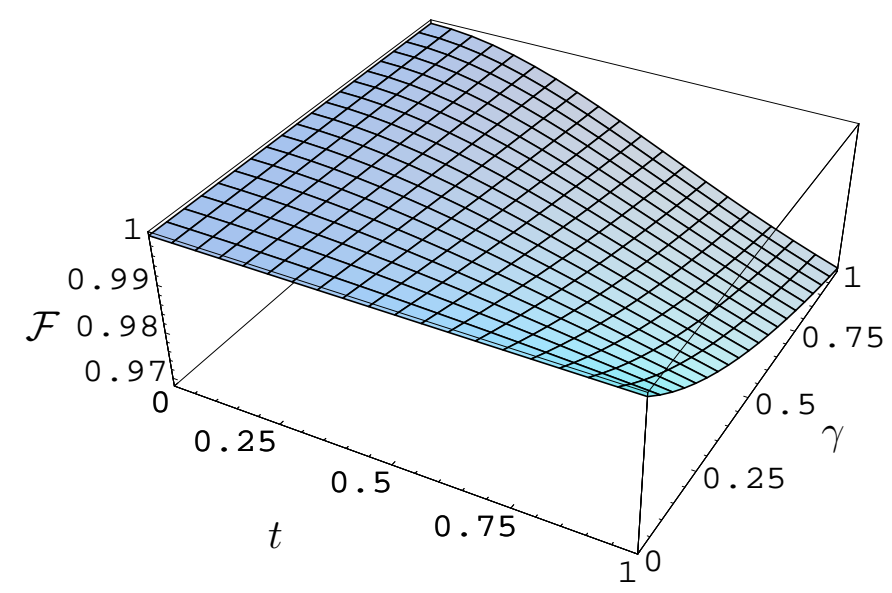

Figure 2: The Fidelity of the tleported sate with $\alpha=0.95$ and $\beta=\sqrt{1-\alpha^{2}}$ The initial state is prepared such that $C_{00}=0.2, C_{01}=C_{10}=0, C_{11}=\sqrt{1-C_{00}^{2}}$ and $\bar{n}=5, \Omega=1$

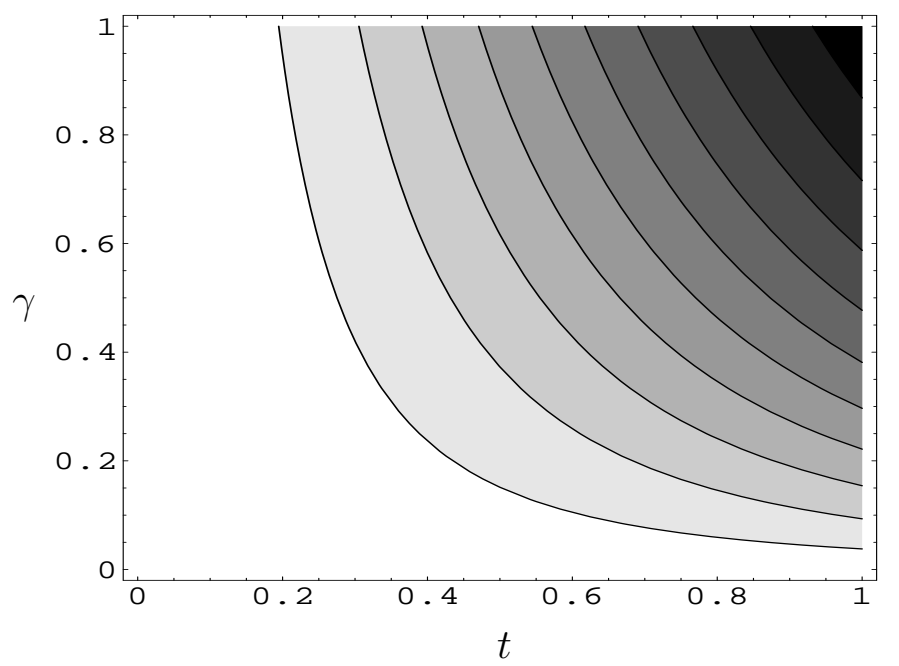

Figure 3: The fidelity, $\mathcal{F}$ of the teleported as contour line against the time $t$ and the chaotic parameter $\gamma$. 
range of time one can teleported the information with unit fidelity for any value of the chaotic parameter.

\section{Conclusion}

Usually sudden death of Entanglement is associated with the environmental effects. It could be radiation field or thermal fluctuations as well. Question what kind consequences nonuniform cavity may have on the quantum information processing was missed yet. Idea of nonuniform cavity (which is of course more realistic from experimental point of view than uniform cavity) implies that constant of coupling between radiation field and optical atom depends on atoms position inside of cavity. This dependence is as a rule ignored for standard Jaynes-Cummings model.

However the nonuniform cavity may lead to the complex dynamic of the system. The aim of the present paper is investigating the consequences of the nonuniform cavity on the quantum information processing. With this in the aim, we studied particular model system to identify new mechanism of decoherence. Key issue is that in our case, in contrast to the common scenario (thermal and environmental effects), sours of decoherence is the initial random parameter over which averaging procedure is done. This parameter itself is a consequence of the random motion of atoms due to nonuniform quantum cavity. Interest to this problem is motivated by the fact that in case of low temperatures, and in the cavities with small mean photon numbers, nonuniform effects on the quantum information processing becomes important, since it recovers thermal and environmental effects.

Basically effect of the random parameter on the degree of entanglement for a twoqubit system and on the fidelity of the teleported state is investigated. The time behavior for both phenomenon, is depicted for the time interval comparable with the correlation length of random parameter. We observe decay of degree of entanglement with time if underling dynamic is chaotic. Decay rate is defined by features of atomic motion inside of cavity and may be scaled along with the correlation length of random variable. Advantage of the offered model is that, decay rate is quite low if initial kinetic energy of the system is small. Also, an important result is that, in spite of de-coherence, minimum degree of entanglement does not reach to zero. This indicates on the possibility, of using given model for performing quantum teleportation with reasonable fidelity, during the large lapse of time.

Acknowledgment: We are grateful to the referee for their constructive com- 
ments and remarks have improve our results. The designated project has been fulfilled by financial support from the Georgian National Foundation (grants: GNSF/STO 7/4-197, GNSF/STO 7/4-179). The financial support of Deutsche Forschungsgemeinschaft through SPP 1285 (contract number EC94/5-1) is gratefully acknowledged by L. Chotorlishvili.

\section{References}

[1] M. A. Nielsen and I. L. Chuang, Quantum Computation and Quantum Information (Cambridge University Press, Cambridge, 2000).

[2] A. Peres, Phys. Rev. Lett. 77, 1413 (1996); R.Horodecki, M. Horodecki and P. Horodecki, Phys. Lett. A 222, 1 (1996).

[3] C. H. Bennett,G. Brassardm C. Crépeau , R. Jozsa, A. Peres and W. K. Wottors, Phys. Rev. Lett.701895 (1993).

[4] A. K. Ekert, Phys. Rev. Lett. 67661 (1991).

[5] C. H. Bennett, G. Brassard, A. K. Ekert. Sci Am. 26950 (1992).

[6] M. Khasin, R. Kosloff, Phys. Rev. A 76012304 (2007).

[7] X. San Ma and An Min Wang, Opt. Commu. 270465 (2007).

[8] Y. Dubi, M. Di Ventra, Phys. Rev. A 79, 012328 (2009).

[9] A. Isar, Int. J. Q. Inf. 6689 (2008); Eur. Phys. J. 160225 (2008).

[10] M. Ikram, Fu-Lili and M. S. Zubairy, Phys. Rev. A75 062336 (2007).

[11] X.-Fa Zhou, Y. Zhang and G.-Gan Guo, Phys. Lett. A363 263(2007).

[12] N. Metwally, I. J. Theor. Physics DOI: 10.1007/s10773-010-0339-9 (2010).

[13] P. Schleich, Quantum Optics in Phase Space (Wiley. VCH, Berlin, 2001).

[14] M. Bin, F. Casagrande and A. Lulli , Laser Phys. 19362 (2009). 
[15] S. V. Prants, M. Edelman,G. M. Zaslavsky, Phys.Rev.E 66, 046222 (2002).

[16] L. Chotorlishvili and Z. Toklikishvili, Eur. Phys. J. D 47433 (2008).

[17] P. Jacquod, P. G. Silvestrov, and C. W. J. Beenakker, Phys. Rev. E. 64, $055203(\mathrm{R})(2001)$.

[18] A. Iomin, Phys. Rev. E. 70, 026206 (2004).

[19] R. A. Jalabert and H. M. Pastawski, Phys. Rev. Lett. 862490 (2001).

[20] F. Cucchietti, C. H. Lewenkopf, E.R. Mucciolo, H. M. Postawski and R. O. Vallejois, Pghys. Rev. Lett. 65046209 (2002).

[21] M. O. Scully and M. S. Zubairy, Quantum Optics (Cambridge University Press, 1997).

[22] K. Zyczkowski, P. Horodecki, A. Sanpera and M. Lewenstein, Phys. Rev. A 58, 883 (1998).

[23] K. furuya, M. C. Nemes and G. Q. Pellegrino, Phys. Ref. ett.80 5524 (1998).

[24] R. Horodeci, M. Horodecki and P. Horodecki, Phys. Lett A222 1(1996).

[25] N. Metwally, M. Abdel-Aty and A.-S. F. Obada, Chaos, Solitons and Fractals 22529 (2004).

[26] M. Riebe, M. Chwalla, J. Benhelm, H. Haeffner, W. Haensel, C. F. Roos and R. Blatt, New J. Phys. 9211 (2007).

[27] M. Tumminello and F. Ciccarello, Eur. Phys. J.160 411 (2008).

[28] Mi-Ra Hwang, D. Park, Jin-Woo Son, S. Tamaryan,J. Phys. A,41 385302 (2008).

[29] S. K. Ozdemir, K. Bartkiewicz, Yu-xi Liu and A. Miranowicz, Phys. Rev. A 76, 042325 (2007).

[30] E. Jung, Mi-Ra Hwang, Y. Hwan, Min-Soo Kim, S.-K.Yoo, H. kim, D. K. Park, J.-W. Son, S. Tamaryan, S.-K. Cha, Phys. Rev. A 78012312 (2008)

[31] N. Metwally, Quantum information procession, DOI: 10.1007/s11128-009-01492 